\section{DIVISION B COMMISSION 50}

\author{
PROTECTION OF EXISTING \\ AND POTENTIAL \\ OBSERVATORY SITES \\ PROTECTION DES SITES \\ ASTRONOMIQUES \\ EXISTANTS OU POTENTIELS
}

\title{
TRIENNIAL REPORT
}

\author{
Richard Green \\ Constance Walker \\ Wim van Driel \\ Elizabeth Alvarez del Castillo, \\ Beatriz Garcia, Margarita Metaxa, \\ Masatoshi Ohishi, Ferdinando Patat, \\ Ramotholo Sefako, \\ Anastasios Tzioumis
}

\section{Introduction}

This will be the last triennial report from Commission 50 under that label, because of the reorganization of the IAU at the end of the triennial period. Fortunately, site protection was recognized as an important ongoing function of the IAU, and the work of the Commission is continuing as Commission C.B7. The Commission has its primary association with Division B because of the technical aspects of its work and association with ground-based facilities, while it has the support of Division $\mathrm{C}$ as an Inter-Division Commission because of the strong need for educating the public on the issues.

The Mission of IAU Commission 50 is to

- Enable and enhance efforts to protect observing sites (for astronomical research) from electromagnetic interference.

- Enable and enhance efforts to protect access to the dark night sky as a cultural heritage for the entire world.

A strong motivator for the public aspect of the mission is IAU Resolution 2009 B5, "In Defense of the Night and the Right to Starlight". It speaks to the night sky as an inspiration, to its scientific and cultural values, to the view of the night sky getting worse, to the need to educate the public, to the need to use intelligent lighting, and to astro-tourism.

Key Excerpts from the Resolution:

An unpolluted night sky that allows the enjoyment and contemplation of the firmament should be considered a fundamental socio-cultural and environmental right, and that the progressive degradation of the night sky should be regarded as a fundamental loss; IAU members [should] be encouraged to take all necessary measures to involve the parties related to skyscape protection in raising public awareness of the educational, scientific, cultural, health and recreational importance of preserving access to an unpolluted night sky for all humankind. 
Protection of the astronomical quality of areas suitable for scientific observation of the Universe should be taken into account when developing and evaluating national and international scientific and environmental policies, with due regard to local cultural and natural values.

The full resolution can be found on page 8 of http://www.iau.org/static/ resolutions/IAU2009_English.pdf

\section{The International Year of Light 2015}

By far the highlight of the triennium was IAU participation in the UN-declared International Year of Light. The stated goal of IYL programs is to promote public and political understanding of the central role of light in the modern world. The founding organization, the European Physical Society, and the Chair of the IYL organization, John Dudley, recognized that astronomy ought to have a voice along with photonics, both for continuing to promote understanding of the way in which we learn about the Universe and of the necessity of quality outdoor lighting to preserve our window to space. He also recognized the programmatic accomplishment of the International Year of Astronomy 2009. He therefore approached IAU General Secretary, Thierry Montmerle, for collaboration and support. As a result of subsequent discussion, astronomy got a highlighted topic for IYL, Cosmic Light. Incoming IAU President, Piero Benvenuti and Thierry Montmerle established an IYL Working Group as an ad hoc working group of the Executive Committee.

The strongest initial effort of the Working Group was to obtain some limited funding from the Executive Committee and to solicit proposals for seed money for internationalscale programs that would be the cornerstone projects of the IAU component of the IYL. Given that there was less than a year's lead-time for astronomy community involvement, the expectation was that most proposals would be building on IYA legacy projects. Twenty-seven strong proposals were received from around the world, including several from developing regions. With oversubscription in excess of $5: 1$, selection criteria needed to be clear, particularly with respect to the goal of having global impact. The following two paragraphs reproduce the project definition and published criteria.

A Cosmic Light cornerstone project is a highlighted astronomy-oriented activity of the IYL with global impact. It must reach across cultural and language boundaries to enhance knowledge of and appreciation for the role of light in observation and measurement of atmospheric and cosmic phenomena and/or for the need for quality lighting to protect the beauty and content of natural light from the sky.

Criteria for endorsement of a cornerstone project include:

- Clear connection to one or more cornerstone themes of the IYL.

- Clear objective for educating, training, and/or informing the defined target audience.

- Demonstrated outcome that the materials and presentation can achieve the objective.

- Clear plan for "training the trainers" in kit use, scientific content, exhibit deployment, etc.

- Demonstration of global scope.

- Clear plan for deployment in multiple geographical regions

- Identification of the major languages in which the material will be presented, along with plans for website, publicity, and other mechanisms for dissemination.

- Well-defined project for development phase, production phase, and deployment, including supportable costing.

- Plausible plan for obtaining or clear description of already contributed support adequate to execute the project at global scale. 
Table 1. The IAU Cosmic Light Working Group

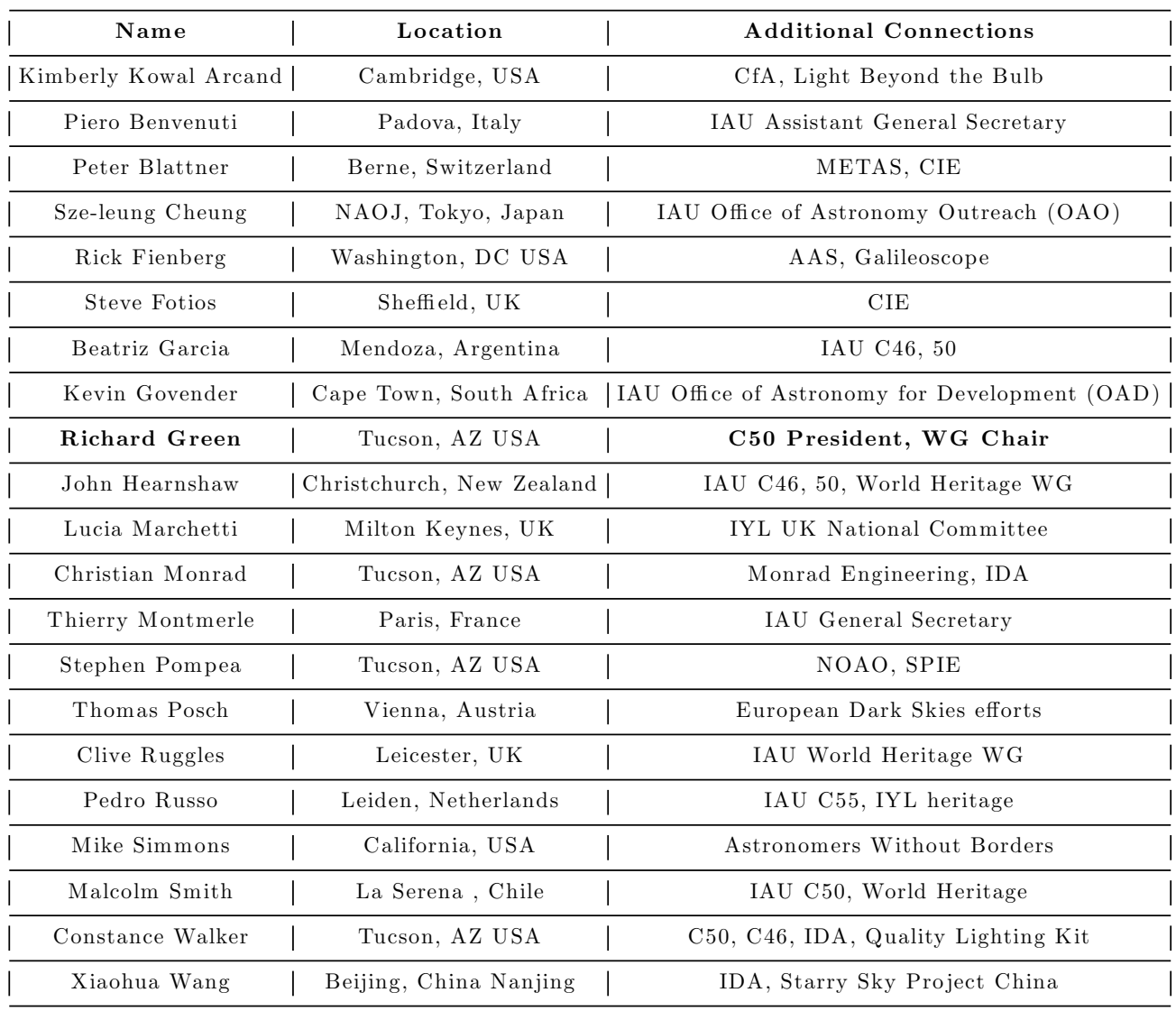

- Metrics for project impact.

- Possible path to sustainability for continuing project activities on a regular basis beyond the IYL.

The Working Group reviewed and ranked the proposals; the top four were selected for seed funding, with the intention of providing them the means to develop additional proposals and prototypes to achieve the broad goals. Those cornerstone projects, as highlighted on the IYL main webpage were Quality Lighting Teaching Kits, Cosmic Light EDU, Light Beyond the Bulb, and Galileoscope. Each is described in turn.

\subsection{Quality Lighting Teaching Kits}

The PI is Constance Walker of NOAO in Tucson, Arizona.

For the International Year of Light in 2015 (IYL2015), the Education and Public Outreach (EPO) Department at the National Optical Astronomy Observatory (NOAO) in the USA has developed a quality lighting education program using their experiences in dark skies awareness, optics and illumination engineering education and public outreach. The hands-on activities in the program will increase public awareness of quality lighting issues through online tutorials, teaching kits and hands-on activities for grades 6-8, but can be expanded to grades 5-10. The activities will focus on a Problem-Based Learning approach, which has students identify the problems and create solutions with some back- 
ground provided. There is a lab on the importance of shielding lights and another on the efficiency of different types of bulbs. These first two labs form the informational basis for a third experience, namely a challenge on lighting for the city of the future.

To help students design the outdoor lighting for the city of the future, students determine what areas of a city need to be lit, what is the optimized footprint on the ground, how many fixtures are needed, what types of lights are most energy efficient, what should be the wattage, what is the required color rendition, what should be the pole height, what should be the intervals between poles, how many hours should the lights be on, etc. In other words, the students must determine how to optimize the lighting plan so as to make it energy efficient, low cost and task-oriented.

The background information that students experience from the first two labs will help them to explore how to build these lights and see how the lights they build perform and to keep iterating. For instance, students could change the height of a pole and see how the footprint of light and its brightness changes on the ground. Students could also compare the calculated energy in a not so good lighting scenario with an improved scenario to conclude how much energy (and cost and carbon footprint) is saved.

The problem-based learning experience begins with an observation of an area of town: what do students see as problematic and what can be done to solve the problem. There might be a globe light lighting everywhere but where it is needed on the ground. Shielding might be surmised as a solution, but what would be the best shield? Something that is full cut off so that the source is not seen? Something that is reflective inside? Situations that illustrate the three main effects of light pollution (glare, light trespass and sky glow) could be illustrated and interacted with, to come up with solutions. Tie-ins to how light pollution affects energy consumption, cost, safety, human health (e.g., sleep deprivation, drops in melatonin levels, etc) and the habits and habitats of wildlife can be discussed as well.

Once these concepts are mastered, the students could possibly be invited to think outside the box in terms of new solutions to lighting in the city of the future: everyone walks around with shoes that are lit; lights in glasses; phosphorescent leaves; new ideas with stored solar energy used at night, etc.

One hundred of the kits were produced and shipped to the collaborators on the project for teacher training and broad use in schools and informal learning settings.

\subsection{Cosmic Light EDU}

The PIs are Rosa Doran (Portugal) and Pedro Russo (Netherlands)

One aspect of the proposal was development of a Dark Sky Meter app for iPhones. It is available at https://itunes . apple.com/app/dsm-lite/id626796278?mt=8

The major aspect is a large set of publicly available educational materials. An overview of that multi-national project follows.

Proposal Partners

NUCLIO Portugal - Coordinator and representative of the Galileo Teacher Training Program:

Leiden University, the Netherlands, Coordinator of Universe Awareness

Astronomical Observatory of University of Valencia Spain

National Optical Astronomical Observatory - US Coordinator of Globe at Night

Southern Illinois University Edwardsville Coordinator of several Outreach programs

European Space Agency HE Space Operations for ESA 
Additional Partners

Inspiring Science Education

Faulkes Telescope

LCOGT

Lawrence Hall of Science

\subsubsection{Resources}

The resources that were developed and are now available to teachers are quoted directly from the Cosmic Light EDU website. Note that a similarly rich variety of activities are found at http://nuclio.org/cosmiclightedukit/activities//

\section{Stellarium}

On this free open source planetarium allows the user to explore cosmic light from a realistic sky in the comfort of your own computer, just like the one we see with the naked eye, binoculars or a telescope.

\section{Celestia}

This free space simulation lets the user explore our universe in three dimensions, travel throughout the solar system, and even beyond our galaxy. Experience at close the objects from which cosmic light one can only have a glimpse of back here on earth.

\section{D Tactile Moon}

This tactile 3D model of the Earth's Moon aims to help blind and visually impaired to learn about the main features on the surface of the Moon. All users will be able to reproduce, by touch, the wonderful experience that the cosmic light reflected from our closest neighbours has on us.

\section{WorldWide Telescope}

This is a rich visualization environment that functions as a virtual telescope, bringing together cosmic light imagery from the best ground- and space-based telescopes to enable seamless, guided explorations of the universe.

\section{Salsa $J$}

This free, student-friendly software allows students to display, analyse, and explore the cosmic light from real astronomical images and other data in the same way that professional astronomers do, making the same kind of discoveries that lead to true excitement about science.

\section{Planetarium Show: The Sky in Your Hands}

This planetarium show is designed for people with visual impairments that makes use of sounds with the support of tactile elements, which can be easily adapted to different types of planetariums creating an image of the Universe as close as possible to the real world.

\section{Extrasolar Planets Lab}

This virtual lab introduces the search for planets outside of our solar system using the Doppler and transit methods. It includes simulations of the observed radial velocities of 
singular planetary systems and introduces the concept of noise and detection enabling to explore the light that reaches us from extra solar planets.

\section{ESA's Fleet Across the Spectrum}

With this poster you'll be able to explain and discover with your students ESA's main astrophysics missions and their observational coverage across the electromagnetic spectrum.

\section{The Sky at Your Fingertips}

Aimed at visually impaired users of all ages who wish to set off on a fascinating journey among the planets, comets and galaxies and discover the wonders within the Universe through images that have been converted into a printing format.

\section{From Earth to the Universe}

Tactile and Braille poster image series accessible to both visually impaired and sighted readers. The materials present celestial objects as they appear through visible-light telescopes and in different spectral regions that are invisible to the naked eye.

\subsection{Light Beyond the Bulb}

The PI is Kimberly Kowal Arcand, Smithsonian Astrophysical Observatory, USA.

'Light: Beyond the Bulb' (http://lightexhibit.org), is a free, open-access international exhibition program on the science of light, launched in January 2015. Along the lines of From Earth to the Universe, it encouraged volunteers to place science in open, accessible locations.

To date, there have been over 650 LBTB exhibits around the world thanks to the dedicated efforts and the creativity of IYL volunteers in over 30 countries, with more than a dozen language translations. Examples of locations include exhibits at the O'Hare Airport in Chicago, U.S., the Village Baykal in Dolna Mitropolia, Bulgaria, the St. Ignatius College Siggiewi Primary School in Siggiewi, Malta, the K11 Art Mall in Shanghai, China, and the Galway Astronomy Festival in Ireland. (See the full exhibit list: http://lightexhibit.org/iylexhibits.html)

'Light: Beyond the Bulb' both has allowed a re-energization of existing networks developed since the International Year of Light 2009, and has provided the opportunity to reach out to new partners and delve into topics that are often beyond the scope of astronomy. For more information, please see our recent article in Communicating Astronomy with the Public: http://www.capjournal.org/issues/18/18_12.php.

Our other effort for IYL 2015 is our new book, Light: The Visible Spectrum and Beyond. The book is a visual exploration of the power and behavior of light, across the electromagnetic spectrum, and how it affects life on earth and everything in the Universe. We were incredibly inspired by the topics of IYL and hope it shows in the work.

\subsection{Galileoscope}

The PI is Douglas Arion of Carthage College, USA.

The Galileoscope (http://galileoscope.org), originally developed for the International Year of Astronomy 2009, solves a long-standing problem: the lack of an optically excellent but inexpensive telescope kit suitable for both optics education indoors and celestial observations outdoors. In the process of assembling the kit, which requires no tools or adhesives, students use the included optical bench to explore how lenses form images and learn about simple lenses, achromatic lenses, positive and negative lenses, 
and other optics concepts. Then, with their completed 50-mm (2-inch) diameter, 25- to 50 -power achromatic refractor (which attaches to any standard photo tripod), they enjoy sharp views of lunar craters and mountains, Jupiter's moons, Saturn's rings, and other bright astronomical objects.

The Galileoscope is an established and successful program for optics and astronomy education, well positioned to contribute to the International Year of Light 2015, especially the themes Science of Light, Light Technology, and Light in Nature. More than 200,000 Galileoscopes are already in use in more than 100 countries, and the continuing influx of orders (most from schools and other institutions doing science education and public outreach) demonstrates ongoing demand. Assembly instructions, observing guides, and standards-based educational materials, including optics-education lesson plans and activities, are freely available in multiple languages. Many are adapted from the US National Optical Astronomy Observatory (NOAO) Hands-On Optics program and as well as from other leading physics-education research programs. Online resources are available on YouTube, Flickr, and other social media.

Among the Galileoscopes distributed so far, about one-third were sold via 30,000 small orders from individuals, and about two-thirds were sold via 500 large orders from institutions. More than 20,000 kits were donated to schools, some in the US and some in poorer countries. For IYL 2015 the growing dealer network handled orders from individual retail customers. The proposers concentrated more on institutional orders and donations in bulk quantities. Galileoscope, LLC, has partnered with the Astrosphere New Media Association to create Telescopes4Teachers (http://telescopes4teachers.org), through which individuals and organizations can make donations (tax-deductible in the US) and designate schools or teachers to receive Galileoscopes.

Many other creative programs did not receive seed money but did get an endorsement from the Working Group. An outstanding example is SkyLight a Global Science Opera (https://kulturped.wordpress.com/prosjekter/prosjekter-under-utvikling/ skylight/). During 2015, schools, universities and art institutions in 35 countries within the Galileo Teacher Training Program (http://www.galileoteachers .org) and Global Hands on Universe (GHOU) collaborated to create and perform a Science Opera inspired by Cosmic Light together, providing a platform for creative science learning as well as cross-border friendship and Cooperation. The concept of SkyLight was presented at the European Planetary Science Congress in Cascais, Portugal, and the Royal Institution in London, England, during Lunar Mission One's event, by PI Oded Ben-Horin. The performance of SkyLight took place in the 35 involved countries simultaneously in October, 2015, and coincided with the final conference of the CREAT-IT project (www.creatitproject.eu).

In addition, many of the ongoing programs initiated during the IYA adapted their themes to the IYL. For example, Globe at Night was considered a citizen-science program of IYL and broke a record this year with over 22,000 observations from 103 different countries. The final meeting(s) of the Working Group and reporting of the Cornerstone Projects will explore the sustainability of those projects and their potential for genuine long-term impact.

\section{Site Protection Issues and Commission Activities}

As was highlighted in the Commission-sponsored Special Session 17 at the 2012 General Assembly in Beijing, the blue light menace of the wholesale conversion to LED outdoor 
lighting continues to grow. As a result of the Special Session, Commission 50 issued three guidelines for quality outdoor lighting (in the IAU Proceedings):

\section{- Full cut-off shielding}

Light emitted just above the horizontal has the most deleterious impact.

\section{- Spectral management to minimize blue-light threat.}

Preservation of spectral access to the night sky in the near zones around research observatories is best achieved with narrow-band amber LEDs, the next generation replacement for low-pressure sodium. The near-term issue is that they do not produce the same level of energy savings as broader-band sources. Urban lighting requirements are different for distant observatories and for the general public in the context of the Resolution. Blue light from urban centers has largely been scattered away before reaching distant research facilities. However, the public's right to a dark night sky requires minimal blue light for outdoor lighting. A conservative figure is limiting the output of a luminaire to no more than $15 \%$ of its radiated energy at wavelengths shorter than $500 \mathrm{~nm}$.

\section{- Zone-appropriate lighting levels, including curfews.}

These principles are in place in both CIE and International Energy Conservation Code guidelines. Astronomers will need to exert downward pressure on the absolute lighting levels prescribed based on zone descriptions.

Countervailing pressures driving public agencies choice of new solid-state luminaires are the desire to conserve energy, a strong consideration particularly in Europe, and the resistance to regulation of commerce, a powerful consideration in the USA. The good news is that there is a new range of commercial LED products with very low output in the blue and ultraviolet. (See the Proceedings of Focus Meeting 21 from the 2015 General Assembly.) Some have correlated color temperatures as low as 2500K, while others have filters that cut off completely below $500 \mathrm{~nm}$. That option for urban areas has progressed more rapidly than increasing the energy efficiency of narrow-band amber LEDs. Protecting observatory sites by replacement of low-pressure sodium lights with narrow-band amber LEDs would require a policy decision by local governments, since energy savings would not be a strong positive factor in the decision.

An important way in which Commission 50 members can influence outdoor lighting standards is by interaction with the CIE, the European based illumination engineering society. Their technical committees consider international standards and safety issues for roadway illumination, appropriate levels of illumination by zone and function, light trespass, and even critical site protection such as observatories and natural areas. Astronomical representation is particularly important, because of our unique understanding of sky brightness impacts and ability to model them, including radiative transfer techniques. Elizabeth Alvarez del Castillo served as the Commission's official representative to the CIE during the report period. She was aided with participation in meetings by Richard Wainscoat (University of Hawaii), David Galadi-Enriquez (CAHA Spain), Javier Diaz Castro (IAC Spain), Ferdinando Patat (ESO) and Ramotholo Sefako (SAAO). Due to changing circumstances of institutional support, Elizabeth completed her term in 2015; she was recognized by the CIE leadership as a highly effective liaison to the organization.

A quantitative assessment of the challenge in control uplighting may soon be in the offing. Presentations by Falchi, Sanchez de Miguel, and others in Focus Meeting 21 at the 2015 IAU GA show the power of new satellite imaging of Earth at night. Combined 
with calibrations from citizen science digital sky monitoring campaigns, they showed the potential for measuring both the temporal changes in sky brightness at observatory sites as well as the mix of lighting sources responsible. Their general conclusion is that there is now no major research facility that is not impacted to some degree by nearby artificial light sources.

The trend is similarly concerning for radio frequency interference. Just as professional OIR observatories are found in increasingly remote sites, radio observatories must rely on the protection of radio quiet zones, established through official policy. They are protected from fixed broadcast sources, but are still subject to cell phone, automobile, and airplane transmissions. Automobile safety systems increasingly contain radio-transmitting elements. Formerly protected astronomical bands in the radio spectrum disappear due to pressure to create more and more commercial broadcast bandwidth for cell phone and other usage (as well as generate revenue in the commercial sale). Commission 50 members, including Liszt, Ohishi, Tzioumis, and van Driel are active in IUCAF, the international commission to protect key radio astronomy frequencies. IUCAF and its national counterparts provide key input for the activities of radio spectrum management.

One way in which Commission 50 aids in site protection is to provide IAU endorsement of sites and regions seeking to obtain recognition or protected status. Commission 50, with IAU Executive concurrence, endorsed the application of the Observatoire du Pic du Midi for International Dark Sky Association Dark Sky Reserve status for the observatory and surrounding area. We also promoted the designation of the area of the AURA Observatories in Chile as an IDA Dark Sky Sanctuary, a new designation targeting regions critical to professional observatories. That activity relates closely to the work of Commission 50 with the former Commission 41 under the leadership of Clive Ruggles to obtain UNESCO World Heritage Status under the Windows to the Universe initiative for the entire group of Chilean professional observatory sites. Begun with the strong drive of Malcolm Smith in Chile, that activity now has the attention of the Chilean government and strong involvement of AURA and other observatory operators. The UNESCO proposal will be structured to accommodate the stated interests of expanding to include the Canary Island observatories and those in Hawaii. Commission 50 agreed to play a dual role in the UNESCO process. One is to provide technical expertise to the proposers to support and validate the aspect of the proposals that cover the unique natural value of their sites to astronomy, particularly in terms of sky brightness and the cooperation of local and national governments in maintaining it. The other is to provide similar expertise to UNESCO itself (with different individuals) to help their evaluation of the same aspects of the proposal. The successors to Commissions 50 and 41 have formed a joint working group to push forward with the UNESCO application process.

\section{Activities Internal to the IAU}

Two major activities occupied Commission 50 during its last year of operations under that title. One was sponsoring a proposal for a Focus Meeting during the 2015 IAU General Assembly. The proposal from PI Constance Walker was accepted as FM 21 under the title "Mitigating Threats of Light Pollution and Radio Frequency Interference". The abstract well expresses the goal of the Commission as well as the purpose of the meeting:

Protecting sites (including observatories and World Heritage sites) through slowing and reversing the encroachment of light pollution requires engaging IAU members and the public on several levels. These actions include producing long-term sky brightness data 
intercomparable with broader public monitoring programs; taking opportunities to educate the public about the value of dark sky preservation; interacting with policy makers and public agencies ranging from localities to the UN to provide legal protection and enforcement for dark sky zones; and interacting with lighting engineers to define dark-sky preserving products and to encourage their deployment. Rapidly advancing solid-state lighting technology and associated promotion is leading to widespread and rapid deployment of blue-rich artificial light sources that threaten to impact a spectral region previously left relatively untouched. Exploring the nature, possible impact, and potential mitigation of this trend is a timely aspect of this session. Similarly, the relentless commercial pressure on use of the broad radio spectrum with the widest possible areal coverage is critical for astronomer attention. We address progress in protection of specific sites, extending legal protection, and advancing the plan for implementing IAU Resolution 2009 B5.

Sixty-one abstracts were submitted, leading to a 2-1/2 day meeting with substantial participation. A detailed description is found in the 2015 GA proceedings. In addition, Commission 50 along with Commission 41 provided a hosted reception at the Bishop Museum in Honolulu on the Sunday evening during the GA. The highlights of the event were the awarding of IDA Dark Sky Preserve status to the AURA Observatories in Chile, the release of a new edition of $\mathrm{N}$ Inoa Hk: Hawaiian and Pacific star names by Rubellite Kawena Johnson, John Kaipo Mahelona, and Clive Ruggles, and a showing of images from the Light Beyond the Bulb exhibit. Over one hundred people, many from the local community participated in the event; commercial sponsors generously provided the refreshments.

The other major activity was related to the restructuring of the IAU. Commission 50 officers and members proposed to continue the site protection work under the new organization of the Union. A key aspect of the proposal are the plans for implementation and the long-term goals:

Implementation:

- Continue the strong voice of astronomy in IYL activities to educate about dark skies and quality lighting

- Sustainable development and provision of information and materials for education and public outreach about dark skies and quality lighting beyond the IYL through an IAU web clearinghouse.

- Creation of regional structure for focusing response on specific issues and providing interface for regional activities.

- Close interaction with CIE to set illumination standards that protect astronomical observations.

- Encouragement of industry efforts to produce spectrally controlled LEDs.

- Education of professional colleagues about urgency of site protection issues; provision of supporting materials for their public presentations. Includes interaction with radio astronomers for site protection against RFI.

- Close interaction with IDA.

- Engagement with the IAU World Heritage activities for astronomical site designation.

- Provision of forum for astronomer efforts to enhance local and national legal frameworks for dark sky protection.

6-Year Goals:

- Set of sustained global activities continuing from IYL (and IYA) initiation. 
- Well populated website with education and outreach materials with global reach.

- Regional structure with points of contact and websites in place.

- Strong IAU participation in CIE Technical Committees and GAs.

- Demonstrated IAU role in promoting amber and blue-blocked LEDs.

- High impact events at IAU GAs in educating about dark skies issues.

- Well-defined relationship with IDA.

- Clear Commission role in UNESCO World Heritage proposals for both preparation and evaluation with respect to dark sky quality of sites.

- Active forums for site protection issues, from legal and public outreach perspectives.

Since the activities of this Commission are both technical and educational in nature, we requested inter-division status to have the benefit of Division $\mathrm{C}$ expertise for education, outreach, and heritage, as well as a strong coupling to the observatories involved in host Division B. The activities of the current C50 and its standing WG on Light Pollution (not the EC IYL WG) overlap so strongly that we propose eliminating the WG and consolidating activities at commission level.

This triennial report is therefore not a retrospective for the end of an era, but rather a progress report on increasingly urgent work. We must engage a growing cadre of our professional peers and sympathetic, informed public to maintain the quality and effectiveness of ground-based professional observing and to work toward achieving the IAU's goal of the right to starlight for the world's population. 